\title{
Thermomechanical Failure Analysis of Through-Silicon Via Interface Using a Shear-Lag Model With Cohesive Zone
}

\author{
Suk-Kyu Ryu, Tengfei Jiang, Jay Im, Paul S. Ho, Fellow, IEEE, and Rui Huang
}

\begin{abstract}
An analytical approach to predict initiation and growth of interfacial delamination in the through-silicon via structure is developed by combining a cohesive zone model with a shear-lag model. Two critical temperatures are predicted for damage initiation and fracture initiation, respectively. It is found that via extrusion significantly increases beyond the second critical temperature. The dependence of the critical temperatures on the material/interfacial properties, as well as the via size (diameter and height), is discussed. In parallel with the analytical approach, finite-element models with cohesive interface elements are employed to numerically simulate the initiation and the progression of interfacial delamination. The numerical results are in good agreement with the analytical solution, and both are qualitatively consistent with reported experimental findings by others.
\end{abstract}

Index Terms-Cohesive zone model (CZM), finite-element analysis (FEA), interfacial reliability, shear-lag model, thermal stresses, thermomechanical, through-silicon via (TSV).

\section{INTRODUCTION}

$\mathbf{T}$ HREE-DIMENSIONAL (3-D) integration has emerged as an effective approach to overcome the wiring limit imposed on chip performance, density and power consumption beyond the current technology node [1]-[6]. Through-silicon vias (TSVs) are important elements for 3-D integration providing direct die-to-die connections to form stacked structures. While the 3-D integration with TSVs offers a promising solution for future technology nodes, serious thermomechanical reliability concerns have been raised [7]-[16]. Due to the coefficient of thermal expansion (CTE) mismatch between the via materials and $\mathrm{Si}$, thermal stresses are ubiquitously induced during processing and thermal cycling of the TSV structures. In some cases, the induced thermal stress is sufficiently high to cause cracking or interfacial delamination of the integrated TSV structures [7]-[10]. In some other cases, the stresses around TSVs may affect the carrier mobility due to the piezoresistivity effect, leading to spatial variation in performance for nearby electronic devices [11]-[13]. Moreover, extrusion of $\mathrm{Cu}$ vias

Manuscript received January 16, 2013; revised March 20, 2013; accepted April 15, 2013. Date of publication May 2, 2013; date of current version March 4, 2014. This work was supported by the Semiconductor Research Corporation.

S.-K. Ryu and R. Huang are with the Department of Aerospace Engineering and Engineering Mechanics, University of Texas, Austin, TX 78712 USA (e-mail: ruihuang@mail.utexas.edu).

T. Jiang, J. Im, and P. S. Ho are with the Microelectronics Research Center, University of Texas, Austin, TX 78712 USA (e-mail: paulho@mail.utexas.edu).

Color versions of one or more of the figures in this paper are available online at http://ieeexplore.ieee.org.

Digital Object Identifier 10.1109/TDMR.2013.2261300

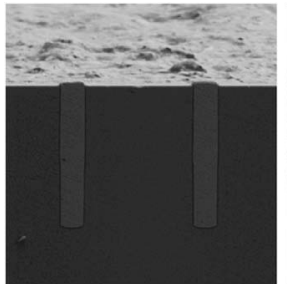

(a)

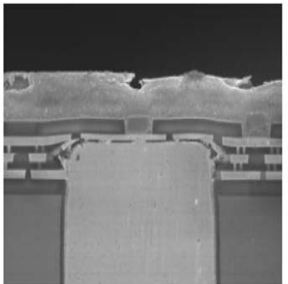

(b)

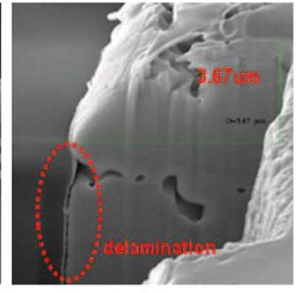

(c)
Fig. 1. Observations of via extrusion and interfacial delamination in TSV structures: (a) Via extrusion after thermal cycling [14]; (b) and (c) Interfacial delamination due to via extrusion [16].

out of Si surface is frequently observed in the TSV structures undergoing high temperature excursion [14]-[17] as shown in Fig. 1. The via extrusion can cause interfacial failure of a TSV and/or cracking in Si near the lower end of TSVs during the thermal processing [16].

To assess the interfacial reliability of TSV structures, we present an analytical approach, which combines a cohesive zone model with a shear-lag model to describe the debonding process. The via extrusion is predicted as a function of the temperature and compared with experimental data. Two critical temperatures are identified from the analytical solution and are compared with finite-element analysis.

\section{Cohesive Zone Model (CZM)}

Cohesive zone models (CZMs) have been widely employed to study the failure processes including nucleation and growth of interfacial cracks [18]-[25]. In the CZM approach, the constitutive behavior of an interface is described by a nonlinear traction-separation law. Various forms of the traction-separation law have been used, such as the perfect plasticity model [22], smooth nonlinear model [23], trapezoidal model [24], and bilinear or triangular model [25]. In the present study, the bilinear traction-separation law (Fig. 2) is employed.

During via extrusion, the interfacial failure is primarily in mode II (shearing mode) [7]. The shear traction at the interface is related to the sliding displacement along the tangential direction by the traction-separation law. Starting from an intact interface, the shear traction first increases linearly with the sliding displacement, with an initial stiffness $K_{0}$. At a critical displacement, the shear traction reaches its maximum, $\tau_{\max }$, after which the shear traction decreases with further sliding due to damage accumulation at the interface. Eventually, the 


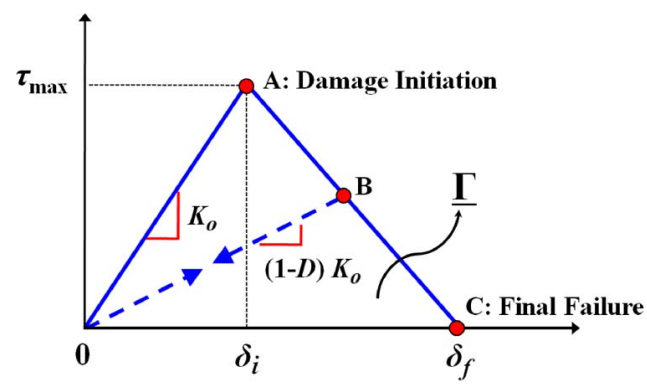

Fig. 2. The bilinear traction-separation law.

shear traction becomes zero at another critical displacement, $\delta_{f}$, after which the traction remains zero as the interface has been fractured. The total work done to fracture the interface is the fracture energy or interface toughness, represented by the area under the traction-separation curve, namely

$$
\Gamma=\frac{1}{2} \tau_{\max } \delta_{f}
$$

The bilinear traction-separation law assumes a linear elastic behavior before the sliding displacement reaches the first critical value, $\delta_{i}=\tau_{\max } / K_{0}$. For $\delta_{i}<\delta<\delta_{f}$, a damage variable, $D$, is defined to describe the damage evolution at the interface through the concept of elastic stiffness degradation [26]

$$
D=\frac{\delta_{f}\left(\delta_{\max }-\delta_{i}\right)}{\delta_{\max }\left(\delta_{f}-\delta_{i}\right)}
$$

where $\delta_{\max }$ refers to the maximum separation attained during the loading history. The damage variable, $D$, evolves from 0 at point $\mathrm{A}$ to 1 at point $\mathrm{C}$ in Fig. 2. In between (point B), the interface is partly damaged $(0<D<1)$, and the shear traction is

$$
\tau=(1-D) K_{o} \delta
$$

Combining (2) and (3) gives that the shear traction decreases linearly with the displacement $\delta$ for $\delta_{i}<\delta<\delta_{f}$

$$
\tau=\tau_{\max } \frac{\delta_{f}-\delta}{\delta_{f}-\delta_{i}} .
$$

During unloading, $\delta_{\max }$ remains constant, and so does $D$. The damage is assumed to be irrecoverable. Therefore, the shear traction decreases linearly as the separation decreases, with the slope $K=(1-D) K_{0}$, as illustrated by the dashed line in Fig. 2.

\section{Shear-LaG Model FOR TSV}

A classical shear-lag model [27], [28] is employed as an analytical approach to predict initiation and propagation of the interfacial cracks in TSV structures. As shown in a previous study [7], the TSV interface may fracture under different modes, depending on the thermomechanical processes (heating or cooling). Here, the focus is on a TSV structure with a positive thermal load $(\Delta T>0)$, for which the interfacial fracture is primarily mode II (shearing mode). For the TSV structures, dielectric oxide and thin barrier layers are commonly deposited

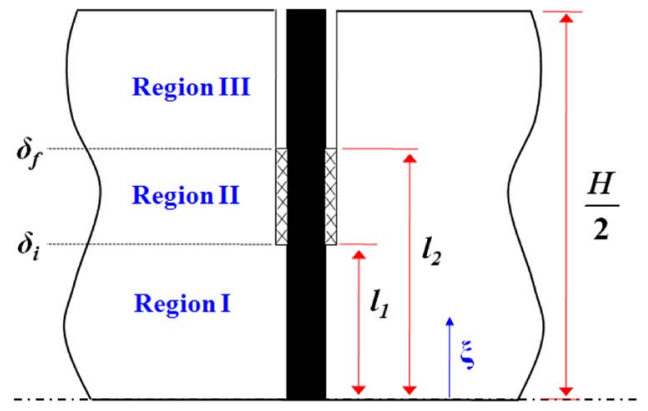

Fig. 3. Schematic of a shear-lag model for a symmetric TSV structure.

on the side wall. These layers are relatively thin (less than $0.4 \mu \mathrm{m}$ ) and thus have little effect on stress distribution in the via and Si. However, those interfacial layers could affect the interfacial properties between the via and $\mathrm{Si}$, which may be taken into account by the parameters in the traction-separation law. In the present study, the oxide and barrier layers are ignored, while the effects of interfacial properties are analyzed.

Consider a TSV structure as shown in Fig. 3. The wafer thickness (or via height) is $H$, and the via diameter is $D_{\text {via }}$. By symmetry, only half of the wafer is considered, with the coordinate $\xi$ measured from the midplane of the wafer. The TSV structure is assumed to be stress free at a reference temperature. As the temperature increases, the CTE mismatch between the via and $\mathrm{Si}$ induces thermal stresses in the via and the surrounding material. The interface between the via and Si first deforms elastically, followed by damage initiation and eventually delamination. The interface may be divided into three regions: intact (I), cohesive (II), and debonded (III). Let $l_{1}$ be the distance from the midplane to the boundary between Region I and Region II, and $l_{2}$ the distance from the midplane to the boundary between Region II and Region III. The interface is initially intact with $l_{1}=l_{2}=H / 2$. As $\Delta T$ increases, a cohesive region first emerges from the free surface, followed by the debonded region. As the two regions grow, $l_{1}$ and $l_{2}$ decrease.

By the shear-lag model, the axial stress in the via $\left(\sigma_{\text {via }}\right)$ is related to the axial displacement $\left(u_{\text {via }}\right)$ as

$$
\sigma_{\mathrm{via}}=E_{\mathrm{via}}\left(\frac{d u_{\mathrm{via}}}{d \xi}-\alpha_{\mathrm{via}} \Delta T\right)
$$

where $E_{\text {via }}$ and $\alpha_{\text {via }}$ are the Young's modulus and CTE of the via material, respectively.

By the equilibrium condition, the shear traction at the interface is

$$
\tau=\frac{D_{\mathrm{via}}}{4} \frac{d \sigma_{\mathrm{via}}}{d \xi} .
$$

By the cohesive zone model, the shear traction is related to the sliding displacement by (3), with the damage variable $D$ taking different values in the three regions. The sliding displacement at the interface is

$$
\delta=u_{\mathrm{via}}-u_{\mathrm{sub}}=u_{\mathrm{via}}-\xi \alpha_{\mathrm{sub}} \Delta T
$$

where $u_{\text {sub }}$ is the axial displacement of the Si wafer, and $\alpha_{\text {sub }}$ is CTE of $\mathrm{Si}$. 
Combining (5)-(7) leads to a single equation for the sliding displacement:

$$
\frac{E_{\text {via }} D_{\text {via }}}{4} \frac{d^{2} \delta}{d \xi^{2}}=\tau(\delta)
$$

where the right-hand side is given by the traction-separation relation of the interface.

\section{A. Stage I: Intact Interface}

In this stage, the entire interface is intact $(D=0)$. Thus, the shear traction is linearly related to the separation,

$$
\tau=K_{0} \delta .
$$

Inserting (9) into (8) leads to

$$
\frac{d^{2} \delta}{d^{2} \xi}=\frac{4 K_{0}}{E_{\text {via }} D_{\text {via }}} \delta
$$

Solving (10) with the boundary conditions: (1) $\sigma_{\mathrm{via}}=0$ at $\xi=$ $H / 2$, and (2) $u_{\text {via }}=0$ at $\xi=0$, we obtain

$$
\delta=\frac{\lambda\left(\alpha_{\mathrm{via}}-\alpha_{\mathrm{sub}}\right) \Delta T}{\cosh \left(\frac{H}{2 \lambda}\right)} \sinh \left(\frac{\xi}{\lambda}\right)
$$

where $\lambda=\sqrt{E_{\text {via }} D_{\text {via }} / 4 K_{0}}$ is a length scale. Correspondingly, the axial stress in the via is

$$
\sigma_{\text {via }}=E_{\text {via }}\left(\alpha_{\text {via }}-\alpha_{\text {sub }}\right) \Delta T\left[\frac{\cosh \left(\frac{\xi}{\lambda}\right)}{\cosh \left(\frac{H}{2 \lambda}\right)}-1\right] .
$$

For the interface to be intact, the maximum separation at $\xi=H / 2$ must be less than $\delta_{i}$. Thus, the critical temperature for damage initiation at the interface is

$$
\Delta T_{c 1}=\frac{\delta_{i}}{\lambda\left(\alpha_{\mathrm{via}}-\alpha_{\mathrm{sub}}\right)} \operatorname{coth}\left(\frac{H}{2 \lambda}\right) .
$$

\section{B. Stage II: Partially Damaged Interface With Regions I and II}

When $\Delta T>\Delta T_{c 1}$, a cohesive region emerges from the surface with $l_{1}<H / 2$. At $\xi=l_{1}$, the sliding displacement $\delta=$ $\delta_{i}$. Thus, in Region I $\left(0<\xi<l_{1}\right)$, the sliding displacement is obtained by solving (10) with the boundary condition at $\xi=l_{1}$, which is

$$
\delta=\frac{\sinh \left(\frac{\xi}{\lambda}\right)}{\sinh \left(\frac{l_{1}}{\lambda}\right)} \delta_{i}
$$

Correspondingly, the axial stress in the via is

$$
\sigma_{\text {via }}=E_{\text {via }}\left[\frac{\cosh \left(\frac{\xi}{\lambda}\right)}{\sinh \left(\frac{l_{1}}{\lambda}\right)} \frac{\delta_{i}}{\lambda}-\left(\alpha_{\text {via }}-\alpha_{\text {sub }}\right) \Delta T\right]\left(0<\xi<l_{1}\right) .
$$

In Region II $\left(l_{1}<\xi<H / 2\right)$, the interface is partially damaged, with the damage variable, $D$, by (2). The shear stress at the interface is given by (4). Inserting (4) into (8), we obtain

$$
\frac{E_{\mathrm{via}} D_{\mathrm{via}}}{4} \frac{d^{2} \delta}{d \xi^{2}}=-A\left(\delta-\delta_{f}\right)
$$

where $A=\tau_{\max } / \delta_{f}-\delta_{i}$.

Equation (16) is solved with the following boundary conditions: (1) $\sigma_{\text {via }}=0$ at $\xi=H / 2$, and (2) $\delta=\delta_{i}$ at $\xi=l_{1}$. Moreover, to determine $l_{1}$, the continuity condition is applied for the axial stress in the via, i.e., $\sigma_{\text {via }}\left(\xi=l_{1}^{+}\right)=\sigma_{\text {via }}\left(\xi=l_{1}^{-}\right)$. These conditions lead to

$\delta=\delta_{f}+C_{1} a \sin \left(\frac{\xi-l_{1}}{a}\right)+C_{2} a \cos \left(\frac{\xi-l_{1}}{a}\right)\left(l_{1} \leq \xi \leq H / 2\right)$

where $C_{1}=\delta_{i} / \lambda \operatorname{coth}\left(l_{1} / \lambda\right), \quad C_{2}=\delta_{i}-\delta_{f} / a, \quad$ and $\quad a=$ $\sqrt{E_{\text {via }} D_{\text {via }} / 4 A}$ is another length scale. The length $l_{1}$ is given implicitly by the following equation:

$C_{1} \cos \left(\frac{H / 2-l_{1}}{a}\right)-C_{2} \sin \left(\frac{H / 2-l_{1}}{a}\right)=\left(\alpha_{\mathrm{via}}-\alpha_{\mathrm{sub}}\right) \Delta T$.

As $\Delta \mathrm{T}$ increases $\left(\Delta T>\Delta T_{c 1}\right), l_{1}$ decreases and the cohesive zone size $\left(l_{\mathrm{cz}}=H / 2-l_{1}\right)$ increases. Eventually, at another critical temperature $\left(\Delta T_{c 2}\right)$, an interfacial crack is initiated with a fully debonded region (Region III) emerging from the free surface. This critical temperature is predicted by setting the sliding displacement at $\xi=H / 2$ to be $\delta_{f}$, namely

$$
C_{1} \sin \left(\frac{H / 2-l_{1}}{a}\right)+C_{2} \cos \left(\frac{H / 2-l_{1}}{a}\right)=0 .
$$

Combining (18) and (19), we obtain an equation for the second critical temperature $\Delta T_{c 2}$

$$
\left(\alpha_{\mathrm{via}}-\alpha_{\mathrm{sub}}\right) \Delta T_{c 2} \sin \left(\frac{H / 2-l_{1}\left(\Delta T_{c 2}\right)}{a}\right)=\frac{\delta_{f}-\delta_{i}}{a} .
$$

\section{Stage III: Partially Fractured Interface With Regions I, II, and III}

When $\Delta T>\Delta T_{c 2}$, an interfacial crack would grow along with a cohesive region, as illustrated in Fig. 3. In Region I $(0<$ $\left.\xi<l_{1}\right)$, the sliding displacement and the axial stress in the via take the same form as for Stage II. In Region II $\left(l_{1}<\xi<l_{2}\right)$, however, the boundary conditions are different from Stage II. By the continuity condition, we have: (1) $\sigma_{\text {via }}=0$ at $\xi=l_{2}$, and (2) $\delta=\delta_{f}$ at $\xi=l_{2}$. The sliding displacement in Region II is then obtained in a similar form

$\delta=\delta_{f}+C_{1} a \sin \left(\frac{\xi-l_{1}}{a}\right)+C_{2} a \cos \left(\frac{\xi-l_{1}}{a}\right)\left(l_{1} \leq \xi \leq l_{2}\right)$.

The continuity conditions at $\xi=l_{2}$ requires that

$$
\begin{aligned}
& C_{1} \cos \left(\frac{l_{2}-l_{1}}{a}\right)-C_{2} \sin \left(\frac{l_{2}-l_{1}}{a}\right)=\left(\alpha_{\mathrm{via}}-\alpha_{\mathrm{sub}}\right) \Delta T \\
& C_{1} \sin \left(\frac{l_{2}-l_{1}}{a}\right)+C_{2} \cos \left(\frac{l_{2}-l_{1}}{a}\right)=0
\end{aligned}
$$


which can be solved simultaneously to determine $l_{1}$ and $l_{2}$, both decreasing with $\Delta T$. The cohesive zone size $\left(l_{\mathrm{cz}}=l_{2}-l_{1}\right)$ remains a constant for $\Delta T>\Delta T_{c 2}$.

In Region III $\left(l_{2}<\xi<H / 2\right)$, the damage variable, $D=1$, and the shear traction $\tau=0$. Thus, this part of the via is not constrained by the $\mathrm{Si}$ wafer and thus deforms freely by thermal expansion. By (8), the sliding displacement increases linearly with $\xi$, i.e.,

$$
\delta=\delta_{f}+\left(\xi-l_{2}\right)\left(\alpha_{\text {via }}-\alpha_{\text {sub }}\right) \Delta T .
$$

Correspondingly, the axial stress in the via is zero for $l_{2}<$ $\xi<H / 2$.

\section{Critical Temperatures for Debonding}

Two critical temperatures are predicted by the cohesive zone model for interfacial failure of the TSV. For the convenience of discussion, the physical and geometrical parameters are normalized as follows:

$$
\begin{aligned}
& \text { Temperature change: } \Delta \bar{T}=\left(\alpha_{\text {via }}-\alpha_{\text {sub }}\right) \Delta T \\
& \text { Interfacial strength: } \quad \bar{\tau}_{\max }=\frac{\tau_{\text {max }}}{E_{\text {via }}} \\
& \text { Interfacial Stiffness: } \quad \bar{K}_{0}=\frac{K_{0} D_{\text {via }}}{E_{\text {via }}} \\
& \text { Interfacial Toughness: } \quad \bar{\Gamma}=\frac{\Gamma}{E_{\text {via }} D_{\text {via }}} \\
& \text { Via height (aspect ratio): } \quad \bar{H}=\frac{H}{D_{\text {via }}} .
\end{aligned}
$$

The first critical temperature defines the temperature change when an initially intact TSV structure starts to accumulate damage at the interface, forming a cohesive zone near the free surface. By (13), the critical temperature after normalization can be expressed as a function of three dimensionless parameters, $\bar{H}, \bar{\tau}_{\text {max }}$, and $\bar{K}_{0}$

$$
\Delta \bar{T}_{c 1}=\frac{2 \bar{\tau}_{\max }}{\sqrt{\bar{K}_{0}}} \operatorname{coth}\left(\bar{H} \sqrt{\bar{K}_{0}}\right) .
$$

Note that the first critical temperature (for damage initiation) is independent of the interfacial toughness, but depends on the interfacial strength and the aspect ratio of the via. In the limiting case for a high aspect ratio, $\bar{H} \gg 1$, we have approximately

$$
\Delta \bar{T}_{c 1} \approx \frac{2 \bar{\tau}_{\max }}{\sqrt{\bar{K}_{0}}} .
$$

The second critical temperature defines the temperature when an interfacial crack starts to nucleate from the cohesive zone with a fully debonded region. By (20), the critical temperature is given implicitly. After normalization, it can be expressed as a function of four dimensionless parameters, $\bar{H}$, $\bar{\Gamma}, \bar{\tau}_{\max }$, and $\bar{K}_{0}$

$$
\Delta \bar{T}_{c 2}=f\left(\bar{\Gamma}, \bar{\tau}_{\max }, \bar{K}_{0}, \bar{H}\right) .
$$
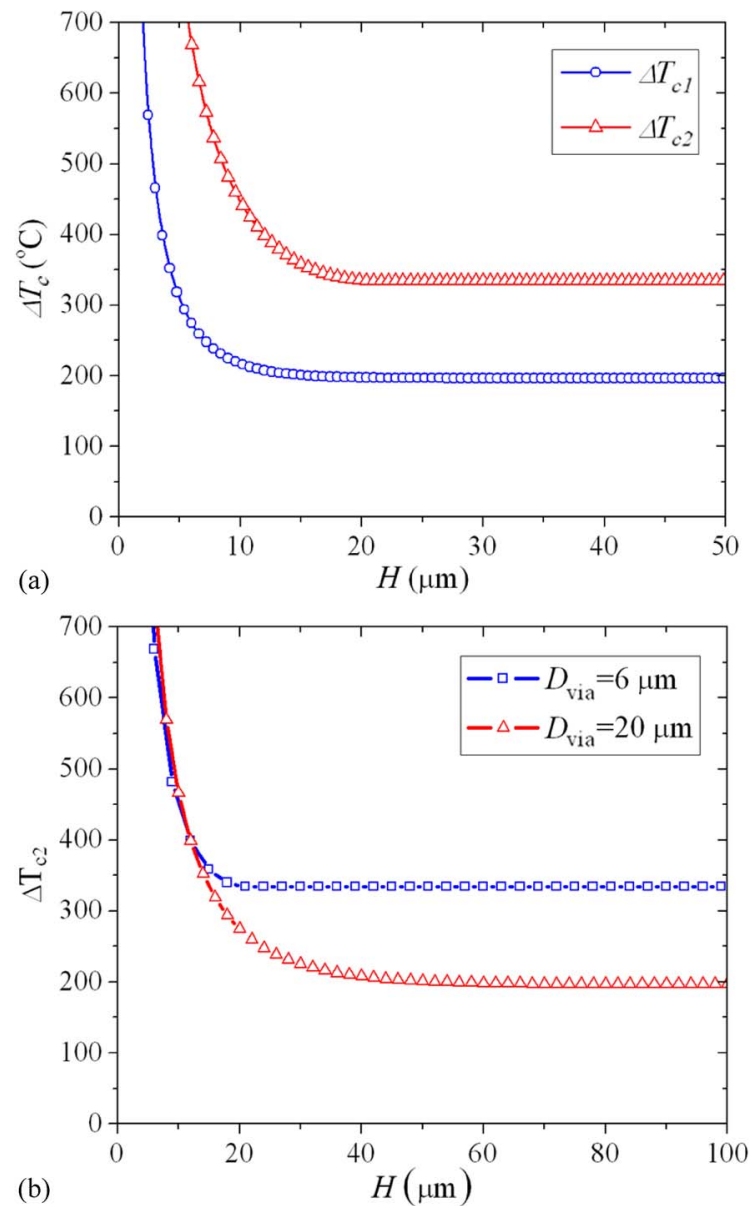

Fig. 4. (a) Critical temperatures for interfacial failure of the TSV structure; (b) Effect of via diameter on the second critical temperature.

Thus, the second critical temperature (for crack initiation) does depend on the interfacial toughness in addition to the other three parameters. In the limiting case for a high aspect ratio, $\bar{H} \gg 1$, we have approximately

$$
\Delta \bar{T}_{c 2} \approx \sqrt{8 \bar{\Gamma}} .
$$

Similar results have been obtained previously for fiberreinforced composites [29].

For an example, both critical temperatures are calculated in this section by using the following properties for the TSV structure with $D_{\text {via }}=6 \mu \mathrm{m}: E_{\mathrm{via}}=110 \mathrm{GPa}, \alpha_{\mathrm{via}}-\alpha_{\mathrm{sub}}=$ $14.7 \mathrm{ppm} /{ }^{\circ} \mathrm{C}, \tau_{\max }=150 \mathrm{MPa}, \Gamma=2.0 \mathrm{~J} / \mathrm{m}^{2}$, and $\delta_{i}=$ $10 \mathrm{~nm}$. The normalized parameters as defined by (26)-(28) are

$$
\begin{aligned}
\bar{\tau} & =0.0013 \\
\bar{K}_{0} & =0.81 \\
\bar{\Gamma} & =3 \times 10^{-6} .
\end{aligned}
$$

The two critical temperatures are plotted in Fig. 4(a) as a function of the via height, $H$. Both the critical temperatures increase as $H$ decreases for the case of relatively small via height $(H<$ $20 \mu \mathrm{m})$. For larger via height, the critical temperatures become independent of $H$. By the approximate solutions in (31) and (33), the two critical temperatures are $196{ }^{\circ} \mathrm{C}$ and $333{ }^{\circ} \mathrm{C}$ for large via heights $(H>20 \mu \mathrm{m})$. It is noted that the normalized parameters, $\bar{K}_{0}$ and $\bar{\Gamma}$, depend on the via diameter $\left(D_{\text {via }}\right)$. As 


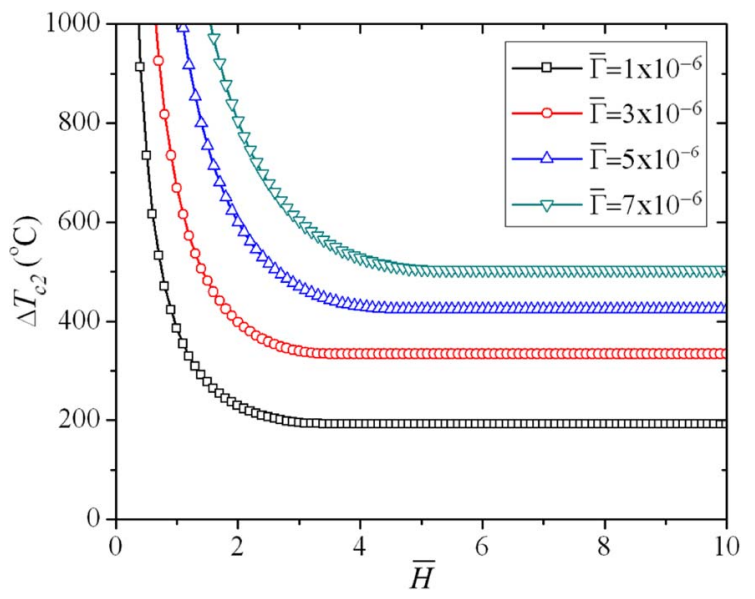

Fig. 5. Effect of interfacial toughness on the critical temperature $\left(\bar{\tau}_{\max }=\right.$ 0.0013 and $\bar{K}_{0}=0.81$ ).

the via diameter increases, $\bar{K}_{0}$ increases and $\bar{\Gamma}$ decreases. As a result, by (31) and (33), both the critical temperatures decrease if the via height $(H)$ remains relatively large. As shown in Fig. 4(b), the second critical temperature merges to a same curve for both via diameters considered when the via height is small $(H<10 \mu \mathrm{m})$, but decreases to a lower level for the larger via diameter when the via height is large. In particular, consider a TSV structure subjected to a thermal load, $\Delta T=300{ }^{\circ} \mathrm{C}$. By Fig. 4(b), interfacial delamination would not occur in the smaller via with $D_{\text {via }}=6 \mu \mathrm{m}$, because $\Delta T<\Delta T_{c 2}$ for all via heights. However, for the larger via with $D_{\text {via }}=20 \mu \mathrm{m}$, interfacial delamination would occur if the via height is greater than a critical value $(H>20 \mu \mathrm{m})$.

The critical temperatures could be much lower for TSVs with larger diameters, as shown in Fig. 4(b). For relatively large via height $(\bar{H} \gg 1)$, both the critical temperatures are inversely proportional to the square root of $D_{\text {via }}$, as predicted by (31) and (33). Furthermore, the effects of interfacial properties, such as toughness and strength on the critical temperatures, were investigated. Fig. 5 shows that the second critical temperature becomes lower as the interfacial toughness decreases. In contrast, the first critical temperature is independent of the toughness as predicted by (30). In a previous study, we observed TSV extrusion due to local plastic deformation in the $\mathrm{Cu}$ vias [14]. Toughness can be enhanced by material plasticity since plastic deformation dissipates energy around the crack tip. Therefore, the second critical temperature would increase in the presence of plastic deformation, and interfacial delamination would be suppressed when $\Delta T<\Delta T_{c 2}$.

The effect of interfacial strength on the critical temperature is shown in Fig. 6. The first critical temperature increases with increasing interfacial strength [Fig. 6(a)]. However, the second critical temperature shows an opposite trend. For a fixed interfacial toughness, the second critical temperatures for different interfacial strengths converge to the same value for sufficiently large aspect ratios $(\bar{H} \gg 1)$, as predicted by (33) [Fig. 6(b)]. For relatively small aspect ratios, the second critical temperature depends on the interfacial strength. By the bilinear tractionseparation relation (Fig. 2), for the same toughness, a higher interfacial strength results in a smaller critical sliding separation $\left(\delta_{f}\right)$ at the final failure. Consequently, the second critical tem-

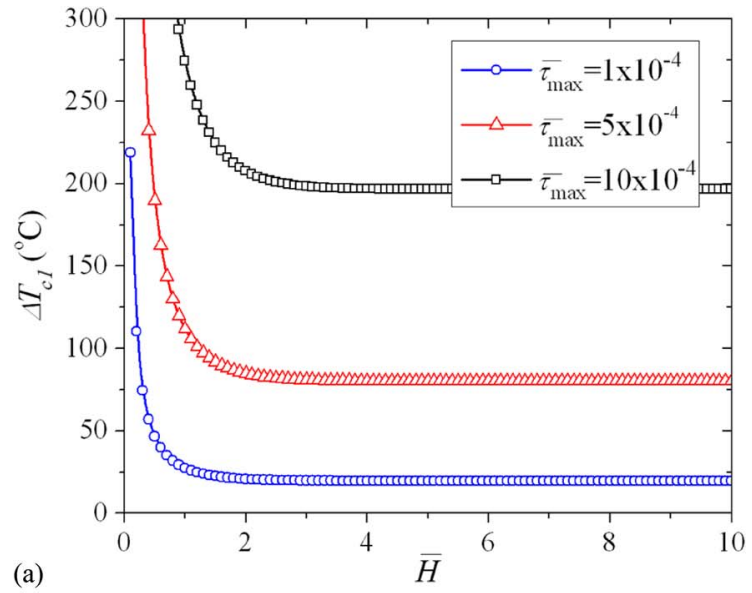

(a)

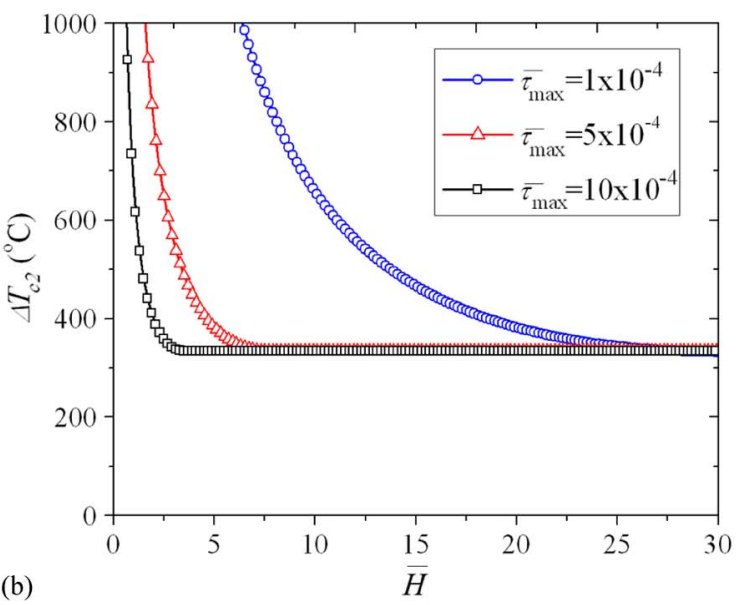

Fig. 6. Effect of interfacial strength on the critical temperatures $(\bar{\Gamma}=3 \times$ $10^{-6}$ and $\bar{K}_{0}=0.81$ ): (a) First critical temperature; (b) Second critical temperature.

perature decreases with increasing interfacial strength. The size of the cohesive zone reaches a steady state when $\Delta T>\Delta T_{c 2}$, which decreases with increasing interfacial strength. If the cohesive zone size is small compared to the via depth (smallscale bridging), the result becomes independent of the strength. However, when the cohesive zone size is comparable to the depth (large-scale bridging), the second critical temperature decreases with decreasing cohesive zone size. Therefore, in general, both the strength and the toughness of the interface are needed to determine the interfacial reliability in the TSV structures.

\section{VIA EXTRUSION}

The sliding displacement at the wafer surface may be observed as via extrusion. By the combination of the cohesive zone model and the shear-lag model, the sliding displacement at the surface $(\xi=H / 2)$ is determined as a function of the thermal load $(\Delta T)$. In Stage I $\left(\Delta T<\Delta T_{c 1}\right)$, by (11), the extrusion displacement is

$$
\hat{\delta}=\lambda\left(\alpha_{\text {via }}-\alpha_{\text {sub }}\right) \Delta T \tanh \left(\frac{H}{2 \lambda}\right) .
$$

In this stage, since the interface remain intact, the extrusion displacement depends on the elastic properties of the via and 


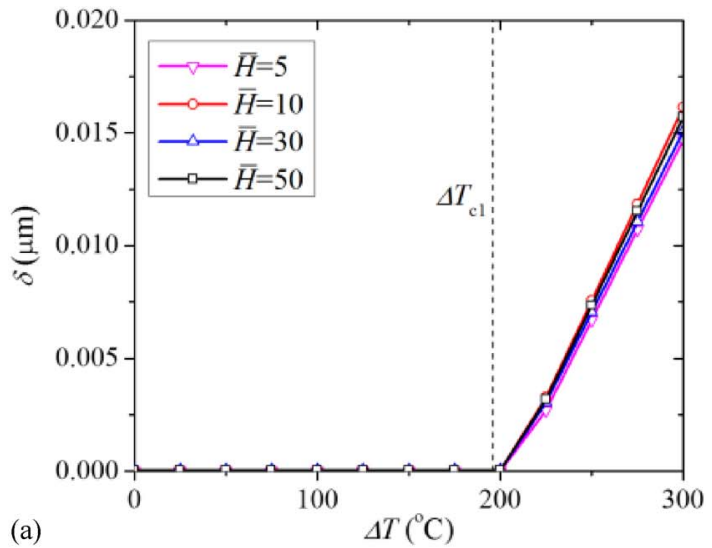

(a)

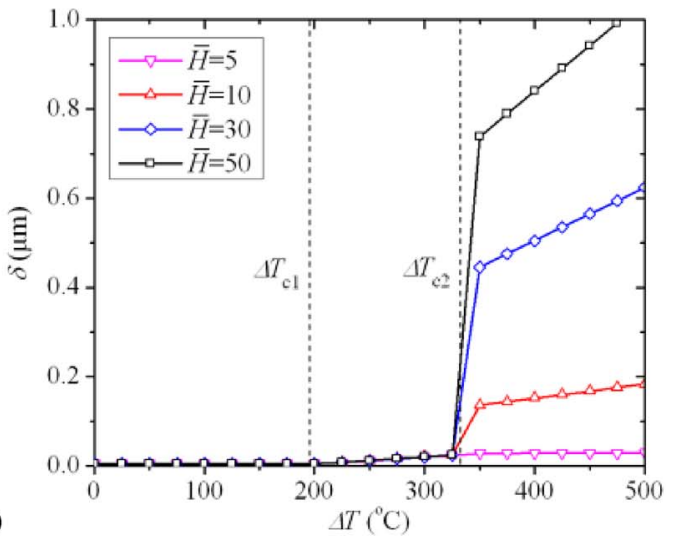

(b)

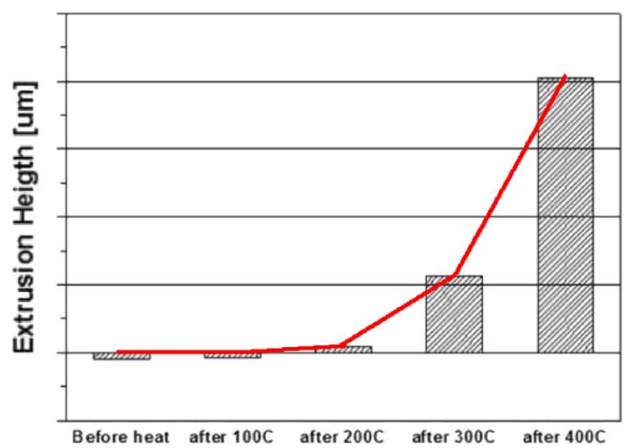

(c)

Fig. 7. Via extrusion displacement for various aspect ratios (a) after first critical temperature; (b) after second critical temperature by the shearlag model; (c) via extrusion observed for various annealing temperatures (Source: [16]).

interface only, which is relatively small $\left(\hat{\delta}<\delta_{i}\right)$. In Stage II $\left(\Delta T_{c 1}<\Delta T<\Delta T_{c 2}\right)$, the extrusion displacement at the surface is given by

$$
\begin{aligned}
\hat{\delta}=\delta_{f}-\left(\delta_{f}-\delta_{i}\right) & \cos \left(\frac{H / 2-l_{1}}{a}\right) \\
+ & \frac{\delta_{i} a}{\lambda} \operatorname{coth}\left(\frac{l_{1}}{\lambda}\right) \sin \left(\frac{H / 2-l_{1}}{a}\right)
\end{aligned}
$$

where $l_{1}$ is a function of $\Delta T$ by (18). Finally, in Stage III $\left(\Delta T>\Delta T_{c 2}\right)$, the extrusion displacement is

$$
\hat{\delta}=\delta_{f}+\left(\frac{H}{2}-l_{2}\right)\left(\alpha_{\text {via }}-\alpha_{\text {sub }}\right) \Delta T
$$

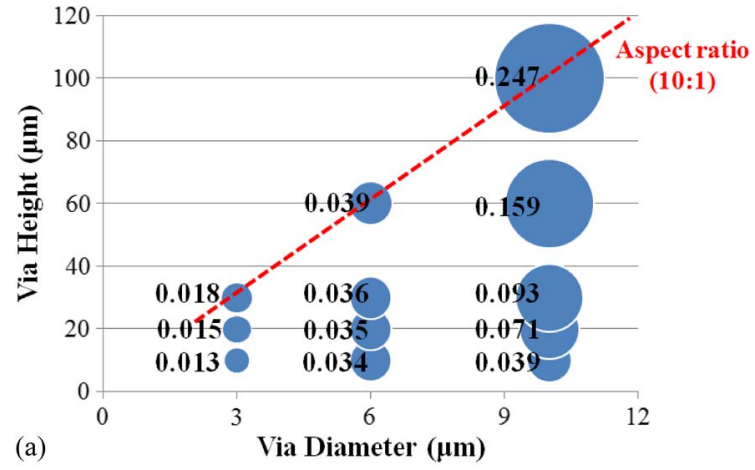

(a)

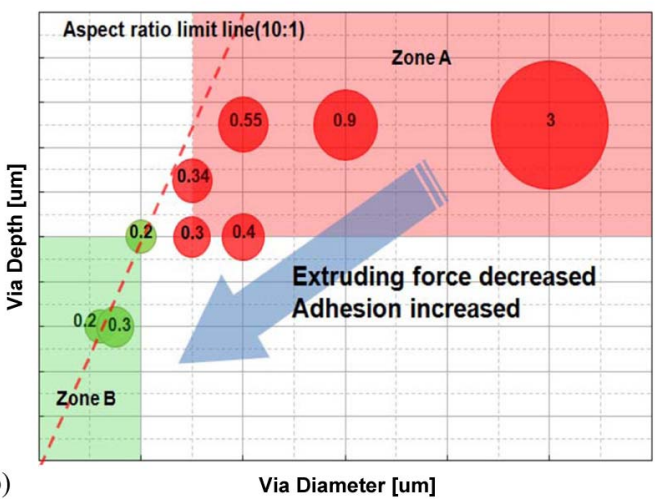

Fig. 8. (a) Via extrusion for various via heights and diameters; (b) Experimental observation of via extrusion and interfacial delamination for different via diameters and depths (Source: [16]).

where $l_{2}$ is a function of $\Delta T$ obtainable by solving (22) and (23) simultaneously.

Fig. 7 plots the extrusion displacement as a function of the thermal load. The extrusion displacement increases linearly during Stage I. At the first critical temperature $\left[196{ }^{\circ} \mathrm{C}\right.$ according to the result in Fig. 4(a)], the extrusion displacement kinks up to increase more rapidly in Stage II. At the second critical temperature $\left(333{ }^{\circ} \mathrm{C}\right)$, the extrusion displacement increases abruptly due to crack nucleation at the interface. Similar trend for via extrusion was observed experimentally by Kang, et al. [16], as shown in Fig. 7(c). Furthermore, the extrusion displacement increases with the normalized via height, which suggests that wafer thinning (or reduction of the TSV height) can help reduce via extrusion.

Via extrusion for various via diameters and heights (or depths) is analytically calculated with $\Delta T=300^{\circ} \mathrm{C}$ [Fig. 8(a)]. The numbers in the bubble plot indicate the magnitude of via extrusion (unit: $\mu \mathrm{m}$ ). It was found that serious interfacial delamination could occur for the vias with relatively large diameters and heights. Kang et al. reported experimental data for via extrusion due to interfacial delamination [16]. The experimental trend in the study [Fig. 5(b)] is consistent with the prediction by the analytical model in the present study even though the numbers in the axes were not provided.

\section{Finite-Element Analysis (FEA)}

Finite-element models have been developed using ABAQUS to simulate the initiation and growth of interfacial delamination in the TSV structure. All the constituent materials are assumed 
(a)

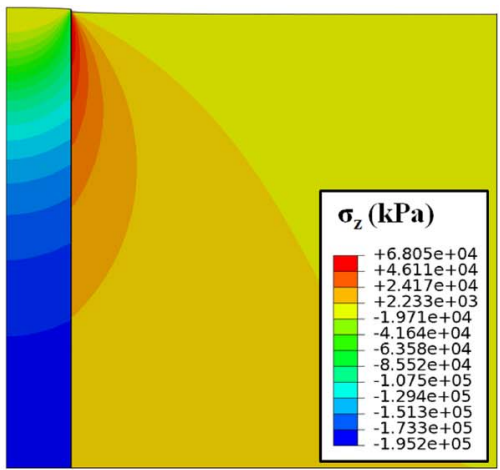

(b)

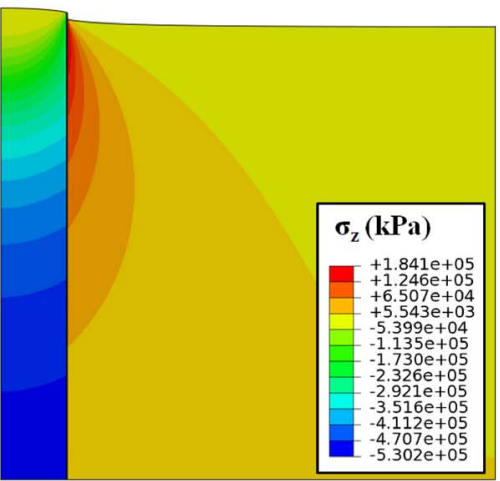

(c)

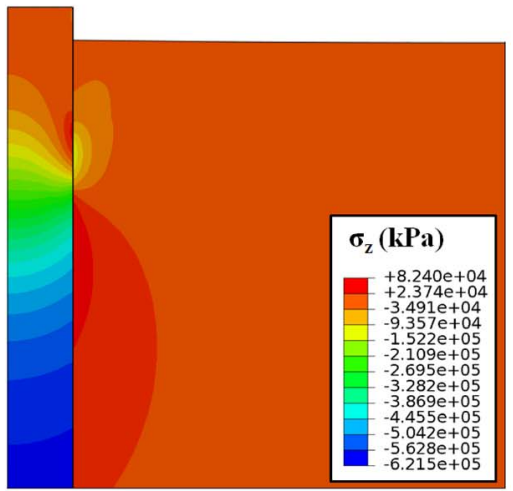

Fig. 9. Simulation of via extrusion during heating process: (a) $\Delta T=100^{\circ} \mathrm{C}$; (b) $\Delta T=280^{\circ} \mathrm{C}$; (c) $\Delta T=350^{\circ} \mathrm{C}$.

to be linear elastic, while the interface between the via and $\mathrm{Si}$ is modeled by cohesive elements with the bilinear tractionseparation relation. In the present study, an axisymmetric FEA model was used for computational efficiency. For the calculation, the material properties listed in (34) were used. The via and $\mathrm{Si}$ parts were meshed with $0.1 \times 0.06 \mu \mathrm{m}^{2}$ axisymmetric element (CAX4R), while the interface was modeled with a layer of cohesive element (COHAX4). Fig. 9 shows the debonding process with increasing temperature. As expected from the analytical solution, the via extrusion dramatically increases after the second critical temperature [Fig. 9(c)].

Fig. 10(a) plots the interfacial damage variable, $D$ along the depth $\left(\mathrm{z} / D_{\text {via }}\right)$ from the FEA model with a fixed height $\left(H / D_{\text {via }}=10\right)$ for different thermal loads. The damage variable remains zero everywhere until the first critical temperature is reached. As predicted by the shear-lag model, the interfacial behavior follows the linear elastic traction-separation relation in Stage I $\left(\Delta T<\Delta T_{c 1}\right)$. For $\Delta T>\Delta T_{c 1}$, a damage zone

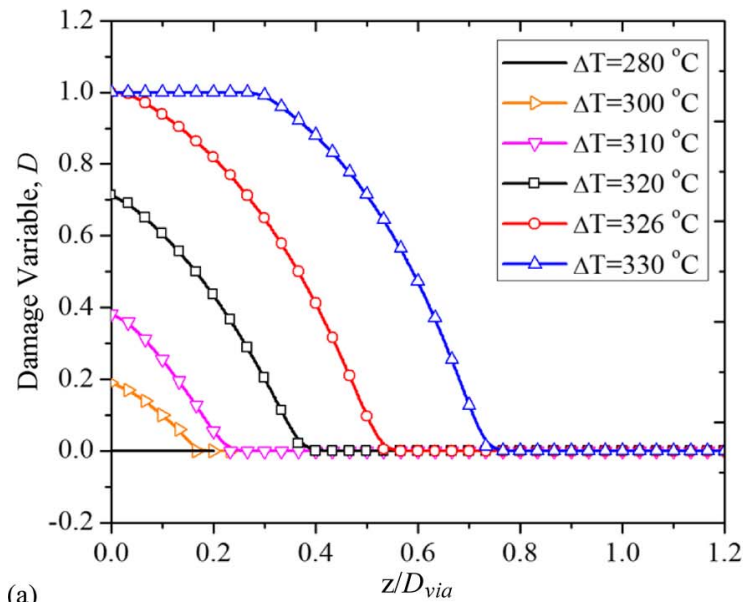

(a)

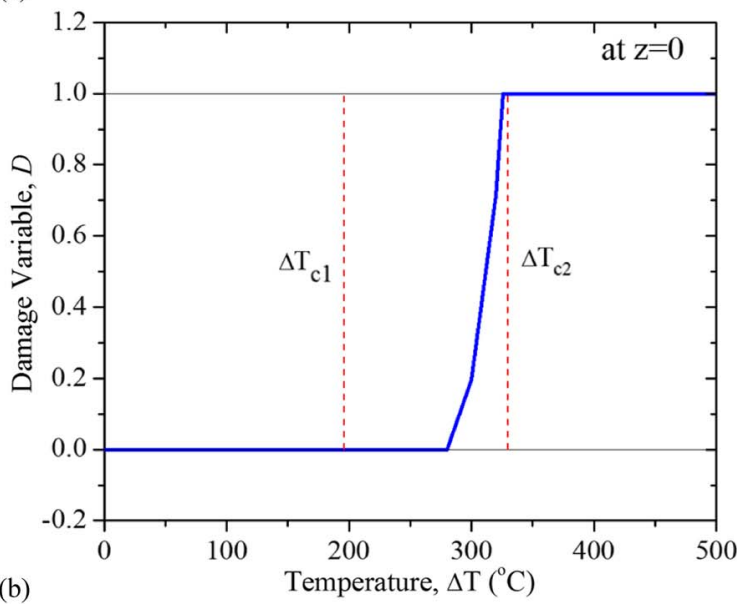

Fig. 10. Damage evolution with increasing temperature, $\Delta T$ : (a) Damage variable vs. depth; (b) Damage variable at $\mathrm{z}=0$ vs. temperature (the red dashed lines indicate the two critical temperatures obtained from the shear-lag model).

emerges with $0<D<1$ and grows along the interface. The maximum damage variable at the surface/interface junction $(\mathrm{z}=0)$ is less than 1 for $\Delta T<326^{\circ} \mathrm{C}$. At the second critical temperature $\left(\Delta T_{c 2}=326^{\circ} \mathrm{C}\right)$, a crack is nucleated with $D=1$ at the surface/interface junction. Subsequently, the crack grows along the interface with a steady-state damage zone ahead of the crack tip. Fig. 10(b) plots the interfacial damage variable at the surface/interface junction $(\mathrm{z}=0)$ as a function of the temperature change, which shows clearly the two critical temperatures. For comparison, the critical temperatures predicted by the shear-lag model are indicated by the vertical dashed lines in Fig. 10(b). Apparently, the first critical temperature is $\sim 200{ }^{\circ} \mathrm{C}$ according to the analytical solution, which is lower than the FEA calculation $\left(\sim 280{ }^{\circ} \mathrm{C}\right)$. On the other hand, the second critical temperature predicted by the shear-lag model is in good agreement with the FEA result. The shear-lag model predicts it to be $333{ }^{\circ} \mathrm{C}$ at $H / D_{\text {via }}=10$, while the FEA model predicts $326{ }^{\circ} \mathrm{C}$. Moreover, Fig. 11 compares the via extrusion displacement obtained from the FEA model with the prediction by the shear-lag model. The differences between the two models can be attributed to the nonuniform stress distribution in the via as shown in Fig. 9.

It is noted that the present study has assumed linear elasticity for the via material and $\mathrm{Si}$ in both the analytical model and 


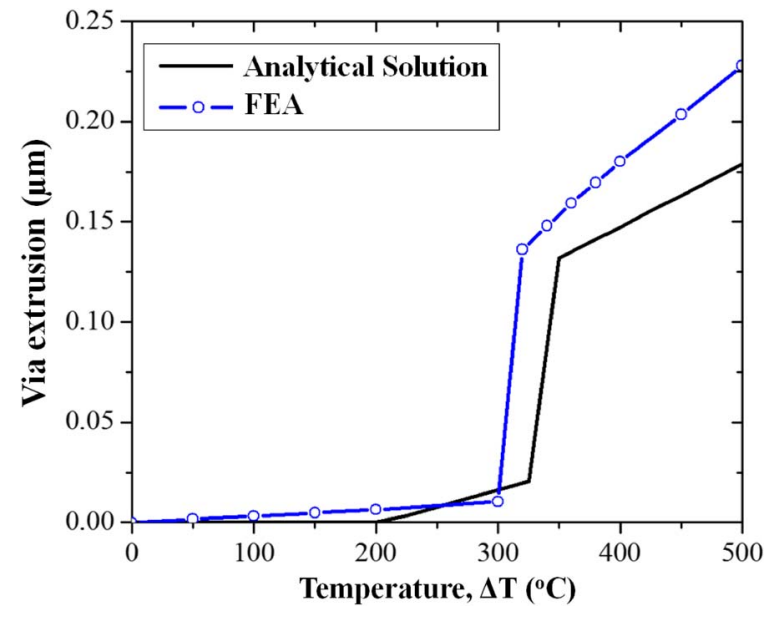

Fig. 11. Via extrusion displacement with increasing temperature.

the FEA analysis. Previous studies [14], [15] have observed inelastic behaviors such as grain growth and plasticity in $\mathrm{Cu}$ vias at high temperatures. In particular, via extrusion may occur as a result of local plastic deformation near the interface without delamination. Nevertheless, by considering interfacial delamination as a potential failure mode of the TSV structure, the results from the analytical model and the FEA analysis are qualitatively in good agreement with experimental observations. Further studies would consider the effects of inelastic material behaviors as well as other failure modes.

\section{SUMMARY}

For the study of the interfacial reliability in TSVs, an analytical approach combining a cohesive zone model and a shearlag model is developed and compared with FEA simulations. Three distinct stages are discussed for the interfacial behavior. Two critical temperatures are predicted for damage initiation and crack nucleation, respectively. The effects of the material parameters and the via dimensions on the critical temperatures and the associated via extrusion are elucidated.

\section{REFERENCES}

[1] The National Technology Roadmap for Semiconductors, Semicond. Ind. Assoc., Washington, DC, USA, 2010.

[2] V. Kripesh, S. W. Yoon, V. P. Ganesh, N. Khan, M. D. Rotaru, W. Fang, and M. K. Iyer, "Three-dimensional system-in-package using stacked silicon platform technology," IEEE Trans. Adv. Packag., vol. 28, no. 3, pp. 377-386, Aug. 2005.

[3] S. F. Al-Sarawi, D. Abbott, and P. D. Franzon, "A review of 3-D packaging technology," IEEE Trans. Compon., Packag., Manuf. Technol. B, Adv. Packag., vol. 21, no. 1, pp. 2-14, Feb. 1998.

[4] M. Karnezos, "3D packaging: Where all technologies come together," in Proc. Int. Electron. Manuf. Technol. Symp. IEEE/CPMT/SEMI, 2004, pp. 64-67.

[5] J. U. Knickerbocker, B. Dang, S. L. Wright, P. S. Andry, E. J. Sprogis, C. K. Tsang, G. S. Patel, L. P. Buchwalter, G. Hua, R. R. Horton, R. J. Polastre, and J. M. Cotte, "3-D silicon integration and silicon packaging technology using silicon through-vias," IEEE J. Solid-State Circuits, vol. 41, no. 8, pp. 1718-1725, Aug. 2006.

[6] W. J. Howell, D. W. Brouillette, J. W. Konejwa, E. J. Sprogis, and S. J. Yankee, "Area array solder interconnection technology for the threedimensional silicon cube," in Proc. ECTC, 1995, pp. 1174-1178.
[7] S. K. Ryu, K.-H. Lu, X. Zhang, J. Im, P. S. Ho, and R. Huang, "Impact of near-surface thermal stresses on interfacial reliability of through-siliconvias for 3-D interconnects," IEEE Trans. Device Mater. Rel., vol. 11, no. 1, pp. 35-43, Mar. 2011.

[8] N. Ranganathan, K. Prasad, N. Balasubramanian, and K. L. Pey, "A study of thermo-mechanical stress and its impact on through-silicon vias," J. Micromech. Microeng., vol. 18, no. 7, pp. 075018-1-075018-13, Jul. 2008.

[9] C. S. Selvanayagam, J. H. Lau, X. Zhang, S. Seah, K. Vaidyanathan, and T. C. Chai, "Nonlinear thermal stress/strain analyses of copper filled TSV (Through Silicon Via) and their flip-chip microbumps," IEEE Trans. Adv. Packag., vol. 32, pp. 720-728, Nov. 2009.

[10] S. K. Ryu, Q. Zhao, M. Hecker, H. Y. Son, K. Y. Byun, J. Im, P. S. Ho, and R. Huang, "Micro-Raman spectroscopy and analysis of near-surface stresses in silicon around through-silicon vias for three-dimensional interconnects," J. Appl. Phys., vol. 111, no. 6, pp. 063513-1-063513-8, Mar. 2012.

[11] S. Thompson, G. Sun, Y. Choi, and T. Nishida, "Uniaxial-process-induced strained-Si: Extending the CMOS roadmap," IEEE Trans. Electron Devices, vol. 53, no. 5, pp. 1010-1020, May 2006.

[12] A. P. Karmarker, X. Xu, and V. Moroz, "Performance and reliability analysis of 3D-integration structures employing Through Silicon Via (TSV)," in Proc. IEEE 47th Annu. Int. Rel. Phys. Symp., 2009, pp. 682-687.

[13] S. K. Ryu, K.-H. Lu, T. Jiang, J. Im, R. Huang, and P. S. Ho, "Effect of thermal stresses on carrier mobility and keep-out zone around throughsilicon vias for 3-D integration," IEEE Trans. Device Mater. Rel., vol. 12, no. 2, pp. 255-262, Jun. 2012.

[14] S. K. Ryu, T. Jiang, K. H. Lu, J. Im, H.-Y. Son, K.-Y. Byun, R. Huang, and P. S. Ho, "Characterization of thermal stresses in through-silicon vias for three-dimensional interconnects by bending beam technique," Appl. Phys. Lett., vol. 100, no. 4, pp. 041901-1-041901-4, Jan. 2012.

[15] T. Jiang, S. K. Ryu, Q. Zhao, J. Im, R. Huang, and P. S. Ho, "Measurement and analysis of thermal stresses in 3D integrated structures containing through-silicon vias," Microelectron. Reliab., vol. 53, no. 1, pp. 53-62, Jan. 2013.

[16] S. Kang, S. Cho, K. Yun, S. Ji, K. Bae, W. Lee, E. Kim, J. Kim, J. Cho, H. Mun, and Y. L. Park, "TSV optimization for BEOL interconnection in logic process," in Proc. IEEE Int. 3DIC, Osaka, Japan, Jan. 31/Feb. 2 , 2012, pp. 1-4.

[17] B. Patti, "3D finds the Road," in Proc. RTI Workshop 3-D Architect. Semicond. Integr. Packag., Burlingame, CA, USA, Dec. 2009.

[18] Q. D. Yang, M. D. Thouless, and S. M. Ward, "Numerical simulations of adhesively-bonded beams failing with extensive plastic deformation," $J$. Mech. Phys. Solids, vol. 47, no. 6, pp. 1337-1353, Apr. 1999.

[19] Y. Wei and J. Hutchinson, "Models of interface separation accompanied by plastic dissipation at multiple scales," Int. J. Fract., vol. 95, no. 1-4, pp. 1-17, 1999.

[20] Q. D. Yang, M. D. Thouless, and S. M. Ward, "Elastic-plastic mode-II fracture of adhesive joints," Int. J. Solids Struct., vol. 38, no. 18, pp. 32513262, May 2001

[21] H. Mei, S. Gowrishankar, K. M. Liechti, and R. Huang, "Initiation and propagation of interfacial delamination in integrated thin-film structures," in Proc. 12th IEEE ITherm, Las Vegas, NV, USA, Jun. 2010, pp. $1-8$.

[22] D. S. Dugdale, "Yielding of steel sheets containing slits," J. Mech. Phys. Solids, vol. 8, no. 2, pp. 100-104, May 1960.

[23] B. N. Cox and D. B. Marshall, "The determination of crack bridging forces," Int. J. Fract., vol. 49, no. 3, pp. 159-176, Jun. 1991.

[24] B. F. Srensen and T. K. Jacobsen, "Determination of cohesive laws by the J integral approach," Eng. Fract. Mech., vol. 70, no. 14, pp. 1841-1858, Sep. 2003.

[25] C. G. Davila, P. P. Camanho, and M. F. De Moura, "Mixed-mode decohesion elements for analysis of progressive delamination," in Proc. AIAA/ASME/ASCE/AHS/ASC Struct. Struct. Dyn. Mater. Conf., 2001, pp. 16-19.

[26] J. H. Lee and G. L. Fenves, "Plastic-damage model for cyclic loading of concrete structures," J. Eng. Mech., vol. 124, no. 8, pp. 892-900, Aug. 1998.

[27] J. A. Nairn, "On the use of shear-lag methods for analysis of stress transfer in unidirectional composites," Mech. Mater, vol. 26, no. 2, pp. 63-80, Sep. 1997.

[28] D. K. Shetty, "Shear-lag analysis of fiber push-out (indentation) tests for estimating interfacial friction stress in ceramic-matrix composites," J. Amer. Ceram. Soc., vol. 71, no. 2, pp. C-107-C-109, Feb. 1988.

[29] J. P. Outwater and M. C. Murphy, "On the fracture energy of unidirectional laminate," in Proc. 24th Annu. Tech. Conf. Reinforced Plastics/Compos. Div., 1969, pp. 1-8. 


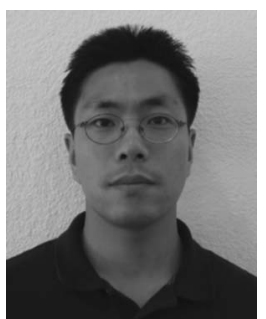

Suk-Kyu Ryu received the B.S. and M.S. degrees in aerospace engineering from Korea Aerospace University, Goyang, Korea, in 1998 and 2000, respectively, and the Ph.D. degree in aerospace engineering and engineering mechanics from The University of Texas at Austin, Austin, TX, USA, in 2011.

He had several years of working experience in the composite material industry, and he was a Researcher in a microsystem laboratory of Korea Institute of Science and Technology, Seoul, Korea. Currently, he is a Senior Engineer at Applied Materials, Sunnyvale, CA, USA. His research interests are in the areas of thermomechanical processrelated reliability issues in electronics materials and microelectronic packaging and coating materials for hardware under corrosive-erosive conditions.

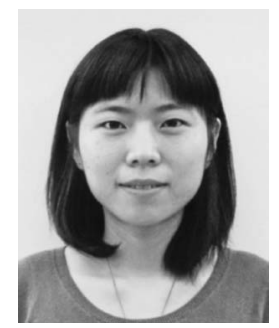

Tengfei Jiang received the B.S. degree in materials science and engineering from Tsinghua University, Beijing, China, in 2006 and the M.S. degree in materials science and engineering from The Ohio State University, Columbus, OH, USA, in 2009. Since 2010, she has been working toward the Ph.D. degree in materials science and engineering at The University of Texas at Austin, Austin, TX, USA.

Currently, her research is focused on the thermomechanical reliability of 3-D interconnects.

Jay Im received the B.S. degree in mechanical engineering from Seoul National University, Seoul, Korea, and the M.S. degree in mechanical engineering and the Doctoral degree in materials science and engineering from Massachusetts Institute of Technology, Cambridge, MA, USA.

$\mathrm{He}$ is a Research Professor in the Laboratory for Interconnect and Packaging, The University of Texas at Austin, Austin, TX, USA. He has more than 130 published papers and 9 U.S. patents. His current research areas are in electronic package and reliability, 3-D through-silicon vias, electromigration of solder joints, and thin-film testing metrology. Up until 2004, he had been with The Dow Chemical Company for 28 years, taking on various R\&D positions including Research Scientist in electronic materials, where he was heading materials science and adhesion efforts for BCB and SiLK dielectrics.

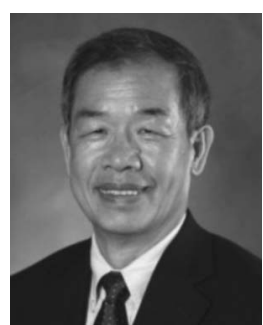

Paul S. Ho (M'91-SM'93-F'02) received the Ph.D. degree in physics from Rensselaer Polytechnic Institute, Troy, NY, USA.

In 1966, he joined the Department of Materials Science and Engineering, Cornell University, Ithaca, NY, where he became an Associate Professor in 1972. In 1972, he joined the IBM T. J. Watson Research Center and became the Senior Manager of the Interface Science Department in 1985. Since 1991, he has been with the faculty of The University of Texas at Austin, Austin, TX, USA, where he was appointed as the Cockrell Family Regents Chair in Materials Science and Engineering and is currently the Director of the Laboratory for Interconnect and Packaging. His current research is in the areas of materials and processing science for interconnect and packaging applications.

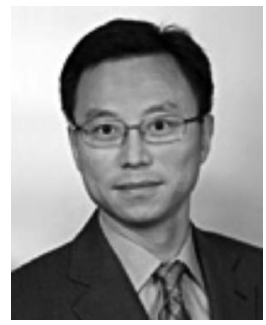

Rui Huang received the B.S. degree in theoretical and applied mechanics from the University of Science and Technology of China, Hefei, China, in 1994 and the Ph.D. degree in civil and environmental engineering, with specialty in mechanics, materials, and structures, from Princeton University, Princeton, NJ, USA, in 2001.

In 2002, he joined the faculty at The University of Texas at Austin, Austin, TX, USA, where he is currently an Associate Professor of aerospace engineering and engineering mechanics and holds the position of Mrs. Pearlie Dashiell Henderson Centennial Fellowship in Engineering. His research interests include mechanics of integrated materials and structures at micro- and nanoscales, reliability of advanced interconnects and packaging for microelectronics, and mechanical instability of thin films and nanostructures. 